\title{
Lossless Visible Watermarking
}

\author{
Shu-Kei Yip, Oscar C. Au, Chi-Wang Ho, Hoi-Ming Wong \\ Department of Electrical and Electronic Engineering \\ The Hong Kong University of Science and Technology \\ Clear Water Bay, Hong Kong \\ Email: \{sukiyip, eeau,jodyho, hoimingw\}@ust.hk
}

\begin{abstract}
The embedding distortion of visible watermarking is usually larger than that of invisible watermarking. In order to maintain the signal fidelity after the watermark extraction, "lossless" property is highlighted in the visible watermarking. In this paper, we propose two lossless visible watermarking algorithms, Pixel Value Matching Algorithm (PVMA) and Pixel Position Shift Algorithm (PPSA). PVMA uses the bijective intensity mapping function to watermark a visible logo whereas PPSA uses circular pixel shift to improve the visibility of the watermark in the high variance region. For the application of medical and military, as they are sensitive to distortion, PVMA and PPSA can be used to insert a visible logo to prevent unauthorized use.
\end{abstract}

\section{INTRODUCTION}

Digital watermarking is one of the ways to prove the ownership and the authenticity of the media. There are mainly two types of watermarking algorithms: visible watermarking and invisible watermarking. For invisible watermarking, the watermark should be perceptually transparent and robustness [1] [2]. For visible watermarking, the watermark should be perceptually visible and robustness. The objectives of visible and invisible watermarks are summarized as table 1.

Table 1. Visible Watermarks V.S. Invisible Watermarks

\begin{tabular}{|l|c|c|}
\hline \multicolumn{1}{|c|}{ Purpose } & Visible & Invisible \\
\hline Validation of intended recipient & --- & $\checkmark \checkmark \checkmark$ \\
\hline Non-reputable transmission & --- & $\checkmark \checkmark \checkmark$ \\
\hline Deterrence against theft & $\checkmark \checkmark \checkmark$ & $\checkmark$ \\
\hline Diminish commercial value without utility & $\checkmark \checkmark \checkmark$ & $\checkmark \checkmark \checkmark$ \\
\hline Discourage unauthorized duplication & $\checkmark \checkmark \checkmark$ & $\checkmark$ \\
\hline Digital notarization and authentication & $\checkmark$ & $\checkmark \checkmark \checkmark$ \\
\hline Identify source & $\checkmark \checkmark \checkmark$ & $\checkmark$ \\
\hline
\end{tabular}

In traditional visible watermarking and invisible watermarking, watermarking is performed by embedding a digital watermark signal into a digital host signal resulting in watermarked signal. Distortion is introduced into the host image during the embedding process and results in Peak Signal-to-Noise Ratio (PSNR) loss. Although the distortion is normally small, some applications, such as medical and military, are sensitive to the embedding distortion and prohibit permanent loss of signal fidelity. This highlights the necessary for lossless/ reversible watermarking, which can recover the original host signal perfectly after the watermark extraction. Most of the existing lossless watermarking algorithms are focus on invisible watermarking [3] [4] [5], however, "lossless" property is more important in visible watermarking than that in invisible watermarking as generally visible watermarking causes greater distortion than that of invisible watermarking.

In this paper, we propose two lossless visible watermarking algorithms. They are Pixel Value Mapping Algorithm (PVMA) and Pixel Position Shift Algorithm (PPSA). PVMA uses the bijective intensity mapping function to watermark a visible logo whereas PPSA uses circular pixel shift to improve the visibility of the watermark in the high variance region.

This paper is organized as follows. In section 2, we present the algorithm of PVMA and PPSA. Section 3 focuses on the security issue of the proposed algorithms. In section 4 , the experimental results are shown. Apart from using PSNR, Weighted Peak Signal-to-Noise ratio (WPSNR) [6] between the original host image and the watermarked image is measured based on the contrast sensitivity function (CFS). In section 5, there is a conclusion.

\section{LOSSLESS VISIBLE WATERMARKING}

We will first introduce the motivations and features of lossless visible watermarking in section 2.1. In section 2.2, the details and the principle of PVMA are described. In section 2.3, we present the details of PPSA.

\subsection{The Motivations and Features of Lossless Visible Watermarking}

There are mainly four reasons (R1-4) why lossless visible watermarking is important, and they are listed as follows:

R1) Visible watermarking is the easiest ways to prove ownership. People can notice the logo without the help of special software.

R2) Embedding distortion in visible watermarking is usually larger than that in invisible watermarking, so "lossless" property is used to ensure the signal fidelity

R3) For the application of military and medical, distortion is not allowed. By using lossless watermarking the original host image can be recovered perfectly after the watermark extraction process.

R4) Some providers want the media with a visible logo or noise for free, and provide the unmarked image or media for fee. 
The features of lossless visible watermarking can be generalized as following points (F1-3):

F1) The regions with uniform intensity are more sensitive to the noise than the regions with non-uniform intensity. As a result, less strength of the watermark is needed for the region with low variance (smooth region) and more to the region with high variance (texture region).

F2) Human visual system (HVS) is more sensitive to the noise in mid-intensity region than that in low intensity region and high intensity region.

F3) The watermark should be embedded in multiply locations, or center position, in order to prevent cropping.

As the logo is visible, the original watermark is available and helps for the recover of the original host image from the watermarked image.

\subsection{Pixel Value Mapping Algorithm (PVMA)}

For an image, $P$, with the size of $M \times N$, the binary watermark $W$, is embedded into $P$ and results in the watermarked image, $Q$, by using the bijective intensity mapping function, $f$. As $W$ is a binary watermark and with the same size as $P, W(x, y) \in\{0,1\}, x=$ $1 \ldots \ldots M, y=1 \ldots \ldots N$,

$$
Q(x, y)= \begin{cases}f(P(x, y)), & \text { if } \quad W(x, y)=0 \\ P(x, y), & \text { if } \quad W(x, y)=1\end{cases}
$$

In this paper, we propose two classes of bijective function, they are linear mapping and piecewise linear mapping.

$$
\begin{aligned}
& \text { 1) Linear Mapping } \\
& \qquad \begin{array}{l}
f(t)=255-t \\
f(t, c)=(t+c) \bmod 256
\end{array}
\end{aligned}
$$

\section{2) Piecewise Linear Mapping}

$$
f(t)=\left\{\begin{array}{lr}
t, & t>T_{1} \text { or } t<T_{2} \\
T_{2}+T_{1}-t, & T_{1} \geq t \geq T_{2}
\end{array}\right.
$$

where $t$ is the pixel value of $P$ and $t \in\{0 \ldots \ldots 255\}$

$$
\text { and } 255 \geq T 1 \geq T 2 \geq 0
$$

$c$ is a user defined constant. We can interpret the intensity mapping function as the rotation of a circle. The intensity value is evenly distributed on the circle. The positive sign means rotation in the clockwise direction whereas the negative sign means rotation in the anti-clockwise direction. The circular representation of equation (2) and (3) are shown in figure 1.
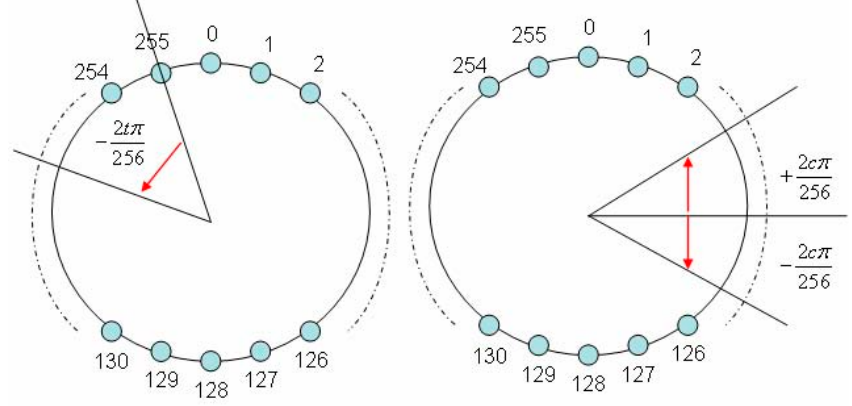

Figure 1. Circular Representation of Equation (2) (Left) and (3) (Right)

By using (2) or (3), the binary watermark can be embedded into the host image visibly. However, for equation (2), it is not favor for large intensity value or small intensity value as large distortion is introduced and for (3), the "salt-and-pepper" artifact appears due to the module operation. In order to reduce the "saltand-pepper" artifact, piecewise linear mapping is proposed. The intensity values near " 0 " and " 255 " are not used; as a result, there is no jumping between " 0 " and " 255 ". For other intensity regions, equation (2) or (3) can be used (equation (4) is a piecewise linear example of using (2)). By partitioning the intensity values into finer regions, the visual artifact can be further reduced as the jumping gap between $T_{1}$ and $T_{2}$ is reduced. Another method is to use the alternative mapping approach. The positions of intensity values on the circle are particular located so that all neighbors are chosen to a closer value, and jumping between " 0 " and " 255 " are prevented. The circular representation of the alternative mapping approach is shown in figure 2 .

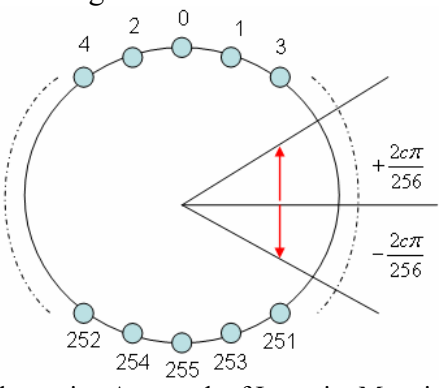

Figure 2. Alternative Approach of Intensity Mapping Function

The major disadvantage of using alternative mapping approach is that the visibility of the watermark is degraded. From the figure 2 , we can see that the pixel values in the left semi-circle are from 1 to 253 (ascending odd order) in clockwise direction whereas that in right semi-circle are from 254 to 2 (descending even order). In the other words, pixels in same smooth region may increase or decrease according to the pixel value is "even" or "odd". The result of using alternative mapping method is shown in figure 6 and 7 .

As $f$ is a bijective function, the inverse of $f, f^{-1}$, exists. As a result, the host image can be recovered with the help of the logo. Let the recovered host image denotes as $R$.

$$
R(x, y)= \begin{cases}f^{-1}(Q(x, y)), & \text { if } \quad W(x, y)=0 \\ Q(x, y), & \text { if } W(x, y)=1\end{cases}
$$

\subsection{Pixel Position Shift Algorithm (PPSA)}

PVMA treats the low variance regions and the high variance regions as the same. This is not favor to the texture region as more energy is needed to ensure the visibility of the watermark in the texture region. As a result, Pixel Position Shift Algorithm (PPSA) is proposed. Apart from using the intensity mapping function described in section 2.2, a circular pixel shift in spatial domain is needed. The circular pixel shift by 1 pixel distance is shown in figure 3 .

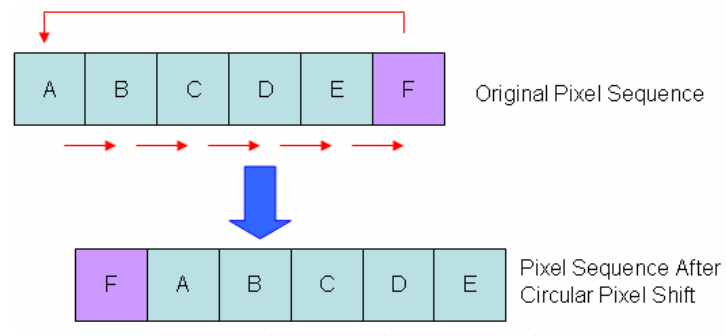

Figure 3. Circular Pixel Shift by 1 Pixel Distance 
For the watermark region, $W\left(x_{1}, y\right)$ to $W\left(x_{2}, y\right)$, with consecutive " 0 "s, a circular pixel shift, $g$, is performed by shifting the pixel to the right with several pixel distances, $d$. The function of $g$ is described as follows:

$$
\begin{array}{r}
g(x, y, d)=P\left(\left(x-x_{1}+d\right) \bmod \left(x_{2}-x_{1}+1\right)+x_{1}, y\right) \\
\text { where } x=\{x 1 \ldots x 2\}
\end{array}
$$

As a result, the overall PPSA representation is

$$
Q(x, y)= \begin{cases}g(f(P(x, y))) & \text { if } W(x, y)=0 \\ P(x, y) & \text { if } W(x, y)=1\end{cases}
$$

For the low variance region, there is nearly no effect after performing circular pixel shift. However, for high variance region, pixel shift means more energy is added. As a result, the logo will be more visible. As circular pixel shift does not destroy the texture pattern, readers can still notice the information of the host image.

As both $f$ and $g$ are bijective, $R$, can be recovered perfectly by applying inverse circular pixel shift followed by inverse intensity mapping function.

\section{DISCUSSION ON SECURITY ISSUE}

In order to enhance the security of the PVMA and PPSA, an enhanced watermarking system with a secret key, $k$, is proposed. A number, which is generated by the secret key, $k$, is added pixel-bypixel during the PVMA/ PPSA using the linear mapping or the piecewise linear mapping approach. Using equation 3 as an example, and it is modified as follows:

$$
\begin{aligned}
f(t, c, n) & =(t+c+n) \bmod 256 \\
& =((t+c) \bmod 256+n) \bmod 256 \\
& =f(f(t, c), n)
\end{aligned}
$$

$n$ is the integer number, which is changed pixel-by-pixel and generated by a secret key, $k$. Form equation (8), we can treat as another PVMA is followed. As $n$ and $k$ are image content independent, the security of the proposed system can be enhanced. The block diagram of watermarking system with $k$ is shown in figure 4.

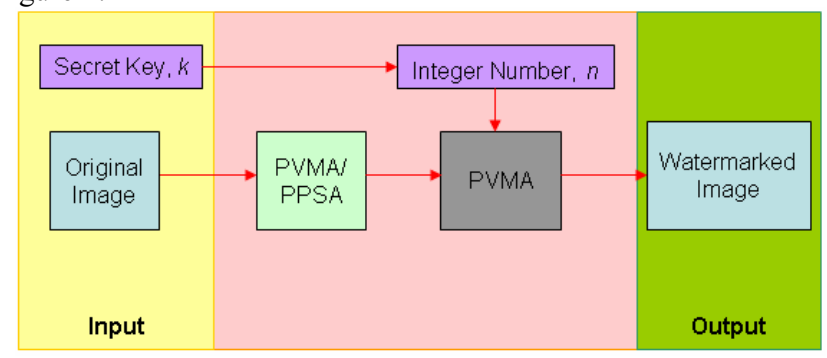

Figure 4. Watermarking System with Secret Key, $k$

\section{EXPERIMENTAL RESULTS}

We have tested the PVMA and PPSA with several standard testing bitmap images. They are Lena, Barbara, Baboon, Pentagon, F16, Fishingboat and Peppers. The original Barbara and the logo are shown in figure 5. The variable, $c$ in (3) is set as 30 and $c$ in alternative mapping method is set as 15 (the pixel value change in both cases are around 30). The watermarked image (Lena) using PVMA and PPSA are shown in figure 6. In figure 7, the rectangular regions shown in figure 5 are used. The top-left rectangular region represents the low variance region whereas the bottom-right rectangular region represents the high variance region. PSNR and WPSNR between the watermarked images and the original host images are used for measuring the visual quality. For
PSNR, Mean Square Error (MSE) between the watermarked images and the original host images is used. For WPSNR, instead of using MSE, Weighted Mean Square Error (WMSE) is used. The weights used in WMSE are based on the contrast sensitive function (CSF) of human visual system [6]. PSNR and WPSNR are used for measuring the visual quality and they are shown in table 2 .

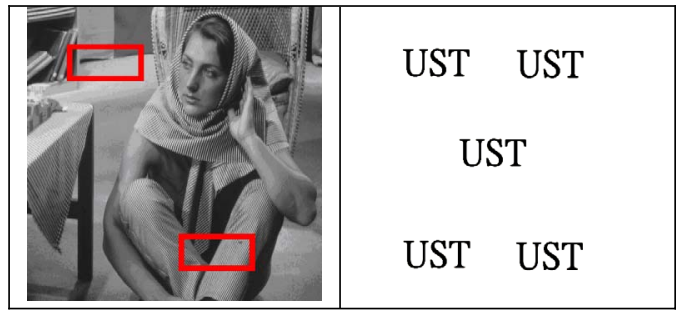

Figure 5. Original Barbara (Left) and Logo (Right)

For PVMA using (2) or PPSA using (2), the PSNR and the WPSNR are both the smallest, it is because large distortion is introduced for small and large pixel values, but the watermark is more visible. From figure 7, the "UST" $\operatorname{logo}$ in bottom-right is more visible in the case of PPSA using (3) than that in PVMA using (3). It is mainly due to the circular pixel shift in PPSA. However, in term of complexity, PVMA is simpler than PPSA, but it treats the low variance region and high variance region in the same way, which is not favor to the texture region. Using alternative mapping method in PVMA, it prevents the jumping between " 0 " and " 255 "; however, it is less visible.

\section{CONCLUSION}

Visible watermarking is one of the ways to prevent illegal use from the unauthorized users by observing the visible logo by human eyes. However, the embedding distortion of visible watermarking is usually larger than that of invisible watermarking. For the application of medical and military, as they are sensitive to distortion, Pixel Value Matching Algorithm (PVMA) and Pixel Position Shift Algorithm (PPSA) can be used to insert the visible logo and the original host image can be perfectly recovered after the watermark extraction.

\section{ACKNOWLEDGEMENT}

This work has been supported by the Innovation and Technology Commission of the Hong Kong Special Administrative Region, China (project no. ITS/122/03 and project no. GHP/033/05).

\section{REFERENCES}

[1] N. Nikolaidis and I.Pitas, "Copyright protection of images using robust digital signatures" Proc. Int. Conf. Acoustics, Speech and Signal Processing, vol. 4, pp. 2168-2171, May 1996

[2] I. Cox, Kilian, T. Leighton, and T. Shamoon, "Secure spread spectrum watermarking for images, audio, and video" Proc Int. Conf. Image Processing, vol. 3, pp. 243-246, Sep. 1996

[3] C. De Vleeschouwer, J.-F. Delaigle, B. Macq, "Circular interpretation of bijective transformations in lossless watermarking for media asset management", IEEE Trans. On Multimeida, vol. 5, no. 1, Mar, 2003

[4] Adnan M. Alattar, "Reversible watermark using the difference expansion of a generalized interger transform", IEEE Trans. On Image Processing, vol. 13, no.8, Aug, 2004

[5] Tian, J., "High capacity reversible data embedding and content authentication", IEEE Int. Conf. On Acoustics, Speech, and Signal Processing, vol. 3, pp. 517-520, April 2003

[6] Makoto Miyahara, Kazunori Kotani, V. Ralph Algazi, “Ojective picutre quality scale (PQS) for image coding”, IEEE Trans. On Communcations, vol. 46, no. 9, Sept 1998 


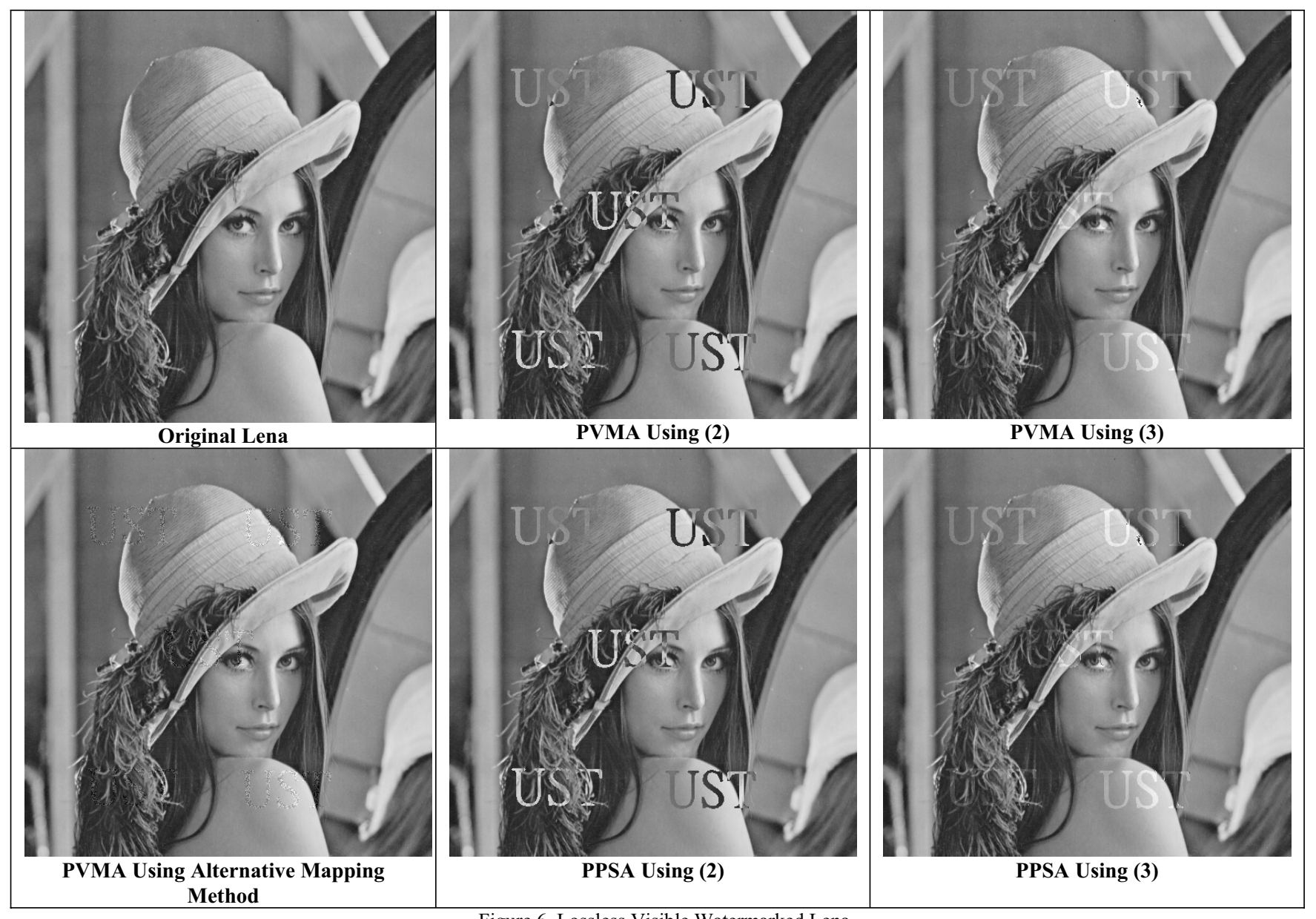

Figure 6. Lossless Visible Watermarked Lena

\begin{tabular}{|c|c|c|c|c|c|}
\hline Algorithm & Top-Left & Bottom-Right & Algorithm & Top-Left & Bottom-Right \\
\hline Original & & $\begin{array}{c}\text { PVMA Using } \\
\text { Alternative } \\
\text { Mapping Method }\end{array}$ & PPSA Using (2) \\
\hline PVMA Using (2) & & & &
\end{tabular}

Figure 7. Lossless Visible Watermarked Barbara

Table 2. PSNR and WPSNR of Different Images

\begin{tabular}{|c|c|c|c|c|c|c|c|c|c|c|}
\hline \multirow{2}{*}{ Images } & \multicolumn{2}{|c|}{ PVMA Using (2) } & \multicolumn{2}{|c|}{ PVMA Using (3) } & \multicolumn{2}{c|}{ PVMA Using Fig. 2 } & \multicolumn{2}{c|}{ PPSA Using (2) } & \multicolumn{2}{c|}{ PPSA Using (3) } \\
\cline { 2 - 12 } & PSNR & WPSNR & PSNR & WPSNR & PSNR & WPSNR & PSNR & WPSNR & PSNR & WPSNR \\
\hline Lena & 22.66 & 28.87 & 33.10 & 39.55 & 33.69 & 48.89 & 22.81 & 28.97 & 31.73 & 38.99 \\
\hline Barbara & 22.73 & 29.20 & 33.63 & 39.75 & 33.70 & 48.72 & 23.08 & 29.29 & 30.77 & 39.25 \\
\hline Baboon & 24.53 & 31.93 & 33.69 & 39.74 & 33.70 & 48.65 & 25.11 & 32.11 & 30.61 & 39.25 \\
\hline F16 & 27.39 & 34.15 & 33.54 & 39.77 & 33.69 & 48.29 & 27.84 & 34.31 & 32.13 & 39.52 \\
\hline Fishingboat & 20.96 & 27.07 & 33.69 & 39.74 & 33.69 & 48.99 & 21.01 & 27.11 & 32.73 & 39.47 \\
\hline Pentagon & 26.24 & 32.59 & 32.73 & 39.69 & 33.70 & 48.62 & 26.51 & 32.76 & 31.94 & 39.42 \\
\hline Peppers & 23.06 & 29.18 & 33.69 & 39.74 & 33.69 & 49.11 & 23.15 & 29.24 & 32.73 & 39.52 \\
\hline
\end{tabular}

